\title{
DENSITY OF GAP STATES DECONVOLUTED FROM PHOTOCONDUCTIVE SPECTRA FOR UNDOPED AMORPHOUS SILICON AND SILICON-CARBON THIN FILMS
}

\author{
T. Pisarkiewicz, T. Stapiński \\ Department of Electronics, Academy of Mining and Metallurgy, Kraków, Poland \\ G. Adriaenssens, W. Lauwerens \\ Department of Solid State and High Pressure Physics, Katholieke Universiteit Leuven \\ Leuven - Heverlee, Belgium \\ AND P. RAVA \\ Elettrorava S.p.A., Savonera Torino, Italy
}

\begin{abstract}
Density of states in amorphous hydrogenated silicon and silicon-carbon thin films were calculated by deconvolution of the optical absorption coefficient $\alpha$ measured by constant photocurrent method. Addition of carbon to silicon lattice increases the optical band-gap and influences the distribution of defect states in the gap.
\end{abstract}

PACS numbers: 73.20.Dx

\section{Introduction}

The principal features of amorphous semiconductors are the short range order, the long range disorder and the coordination defects. The preservation of the short range order results in a similar overall electronic structure of an amorphous material compared to the equivalent crystal. The long range structural disorder originating from the deviations of the bond lengths and bond angles is responsible for the formation of broadened tails of localized states extending into the forbidden gap. Electronic states deep within the gap arise mostly from coordination defects. In a-Si:H these kinds of defects are threefold coordinated silicon dangling bonds in the neutral state $D^{0}$ when singly occupied and in the states $D^{+}$or $D^{-}$when empty or doubly occupied. Positive correlation energy in the reaction of formation $D^{+}$ and $D^{-}$from $D^{0}$ defects ensures that in equilibrium most of defects are in singly 
occupied states $D^{0}$ [1]. Unpaired electrons are paramagnetic and give a strong electron spin resonance (ESR) signal. Amorphous alloy a-Si ${ }_{1-x} \mathrm{C}_{x}: \mathrm{H}$ contains both $\sigma$ and $\pi$ states with defects which may be either $\sigma$ defects (dangling bonds) or $\pi$ defects (abnormal $\pi$-bonded clusters as in a-C:H) [2]. Additional deep states introduced by carbon act as traps for the photogenerated charge carriers lowering the photoconductive response in the alloy as compared to a-Si:H. ESR results do indeed show a strong $x$-related increase in the dangling bond density, which is indicative of the total defect density [3].

The unknown density of states (DOS) is obtained by deconvolution of the absorption coefficient $\alpha(E)$ as proposed for a-Si:H in Ref. [4]. The aim of this paper was to extend the calculations for silicon-carbon alloy with low carbon content and to compare the calculated DOS with that for solar cell grade intrinsic a-Si:H.

\section{Experimental}

The films under investigation were deposited by plasma enhanced chemical vapour deposition (PECVD) method from $\mathrm{SiH}_{4}$ gas phase (a-Si:II) and from $\mathrm{SiH}_{4}$ $+\mathrm{CH}_{4}+\mathrm{H}_{2}$ gas phase (a-Si $\left.{ }_{1-x} \mathrm{C}_{x}: \mathrm{H}\right)$. The multichamber PECVD system based on the cluster tool concept was designed by MV Systems Inc. (Golden, Colorado) and constructed jointly with Elettrorava S.p.A. (Torino, Italy). The deposition conditions for undoped a-SiH were: substrate temperature $200^{\circ} \mathrm{C}$, pressure $0.7 \mathrm{Tr}$, RF power $4 \mathrm{~W}$, silane flow $40 \mathrm{sccm}$, and for $\mathrm{SiC}: \mathrm{H}: \mathrm{SiH}_{4} 5 \mathrm{sccm}, \mathrm{CH}_{4} 3.3 \mathrm{sccm}$, $\mathrm{H}_{2} 100 \mathrm{sccm}$, total pressure $0.6 \mathrm{Tr}$.

The spectral dependence of $\alpha$ in the low absorption region is often calculated from photocurrent measurements. In order to analyze photocurrent quantitatively one has to ensure a constant occupation of gap states with changing photon energy. Measurements with constant photon flux need a special normalization procedure for the photocurrent spectra according to Ref. [5]. In this work the calculations were based on measurements of $\alpha$ in the low absorption region by constant photocurrent method (CPM) [6]. CPM, in contrast to conventional photoconductivity technique, uses varying light intensity to maintain a fixed value of photocurrent as the spectrum is scanned, so that the free-carrier densities (hence the quasi-Fermi level) stay fixed. This technique is not sensitive to surface states and sensitivities well below $\alpha d=1 \times 10^{-5}$ can be achieved ( $d$-thickness of the sample).

\section{Results and discussion}

CPM measures only transitions contributing to the photocurrent, i.e. transitions from valence band states, valence band-tail and deep levels to the non-localized conduction band states (Fig. 1). In the one-electron approximation the absorption coefficient for transitions from extended or localized states with density $N_{i}(\varepsilon)$ to extended states with the density $N_{f}(\varepsilon)$ can be written as [7]:

$$
\alpha(E)=\frac{\text { const }}{E} \int N_{i}(\varepsilon) f(\varepsilon) N_{f}(\varepsilon+E)[1-f(\varepsilon+E)] \mathrm{d} \varepsilon,
$$

where the integration is over all pairs of initial and final states separated by energy $E$ and $f(\varepsilon)$ is the Fermi-Dirac distribution function. The crucial assumption 


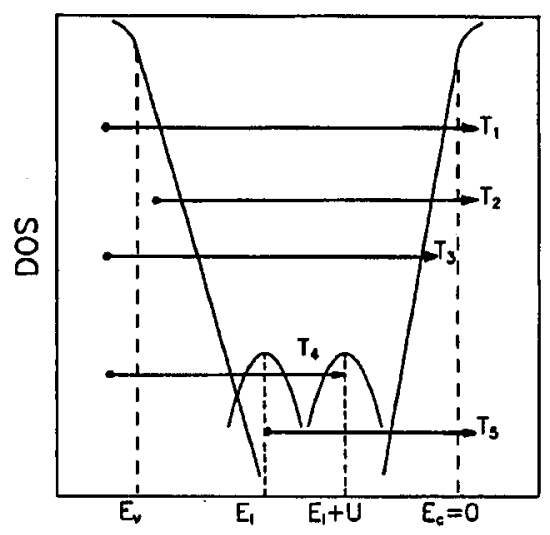

Fig. 1. Main optical transitions in amorphous silicon. Transitions from valence band (VB) to empty $D^{0 /-}$ states $\left(T_{4}\right)$ and from VB to conduction tail CT states $\left(T_{3}\right)$ are essentially not seen in CPM due to the low contribution of holes to the photocurrent. Occupied defect states $D^{0 /+}$ are centred at energy $E_{I}$, empty $D^{0 /-}$ centres are shifted by the correlation energy $U$.
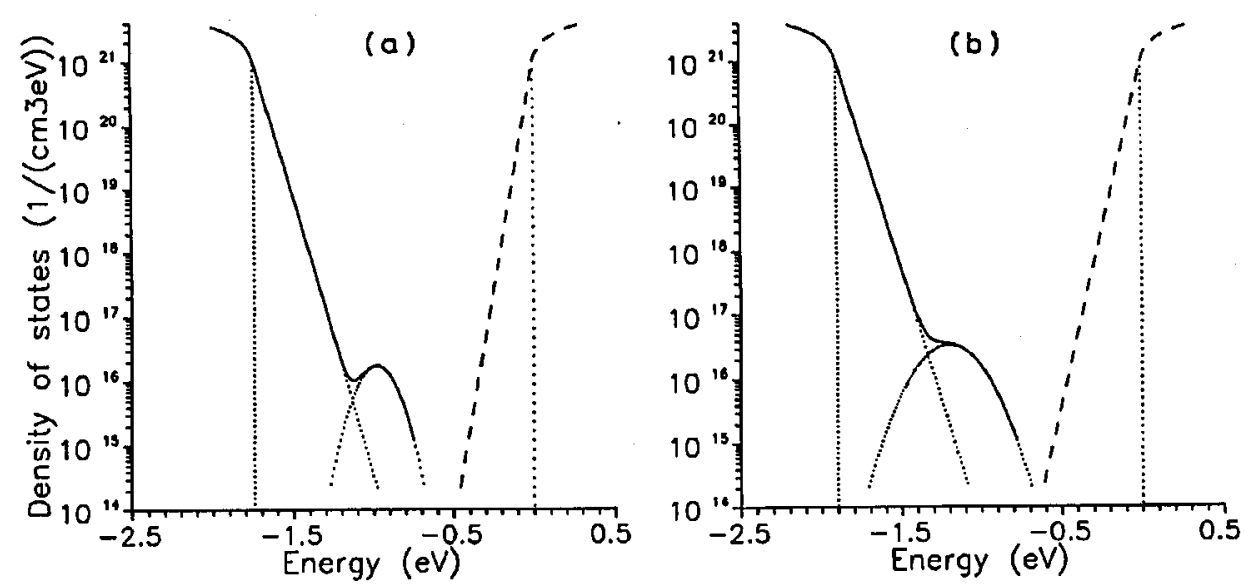

Fig. 2. Density of states deconvoluted from CPM spectra for (a) undoped a-Si:H film (GD) and (b) a-Si $\mathrm{Si}_{0.9} \mathrm{C}_{0.1}: \mathrm{H}$ film (SC8). Full curves are calculated from Eq. (1), dashed curves are drawn assuming the slope of conduction tail $E_{0}^{c}=30 \mathrm{meV}$.

of the above approach is that the transition matrix element is approximately constant and the refractive index is independent of energy. For the valence and conduction bands a parabolic density of states was assumed. For the bandtails the exponentially decaying functions were used and the DOS at midgap was described by a Gaussian distribution. The DOSs deconvoluted from the absorption spectra for undoped solar cell grade a-Si:H (GD) and $a-S_{0.9} \mathrm{C}_{0.1}: \mathrm{H}$ (SC8) are shown in Fig. 2. Comparing Fig. $2 \mathrm{a}$ and $2 \mathrm{~b}$ one can notice that addition of 10 at. $\%$ carbon 
causes the remarkable increase in energy gap (from 1.75 to $1.90 \mathrm{eV}$ ) and also the increase in midgap defect density (from $0.43 \times 10^{16}$ to $1.36 \times 10^{16} \mathrm{~cm}^{-3}$ ). That rather slow increase in defect density for SC8 film can be ascribed to the " $\mathrm{H}_{2}$ dilution" preparation conditions. ESR signal indicates [8] that the defect states in the low carbon alloy $\mathrm{Si}-\mathrm{C}$ are mostly $\mathrm{Si}_{3}^{0}$ states, i.e. silicon dangling bonds. For the composition $x>0.5-0.6 \mathrm{ESR} g$-factor changes abruptly indicating the appearance of $\mathrm{C}$ centres.

Adopting the weak-bond-to-dangling-bond conversion model of Stutzman [9] to the alloys one can account for the reduced defect density in alloys prepared by $\mathrm{I}_{2}$ dilution. The model suggests that defect densities are reduced by making the Urbach tail (the slope of the valence band tail) steeper. In our case the Urbach energy increases from $50 \mathrm{meV}$ for a-Si:H to only $53 \mathrm{meV}$ for a-Si ${ }_{1-x} \mathrm{C}_{x}: \mathrm{II}$.

\section{References}

[1] R.A. Street, Hydrogenated Amorphous Silicon, Cambridge University Press, Cambridge 1991.

[2] J. Robertson, Philos. Mag. B 66, 615 (1992).

[3] F. Demichelis, C.F. Pirri, E. Tresso, Mod. Phys. Lett. B 5, 285 (1991).

[4] M. Vanecek, A. Abraham, O. Stika, J. Stuchlik, J. Kocka, Phys. Status Solidi A 83, 617 (1984).

[5] G. Moddel, D.A. Anderson, W. Paul, Phys. Rev. B 22, 1918 (1980).

[6] M. Vanecek, J. Kocka, J. Stuchlik, Z. Kozisek, O. Stika, A. Triska, Solar Energy Mater. 8, 411 (1983).

[7] N.F. Mott, E.A. Davies, Electronic Processes in Non-Crystalline Materials, Clarendon Press, Oxford 1979, Ch. 6.

[8] F. Demichelis, G. Crovini, F. Giorgis, C.F. Pirri, E. Tresso, G. Amato, H. Herremans, W. Grevendonk, P. Rava, J. Non-Cryst. Solids 164-166, 1015 (1993).

[9] M. Stutzman, Philos. Mag. B 60, 531 (1989). 